ARTICLE

DOI: $10.1038 / \mathrm{s} 41467-018-04713-8$

\title{
Boosting hot electron flux and catalytic activity at metal-oxide interfaces of PtCo bimetallic nanoparticles
}

Hyosun Lee ${ }^{1}$, Juhyung Lim² ${ }^{2}$ Changhwan Lee ${ }^{1,2}$, Seoin Back², Kwangjin An (1) ${ }^{3}$, Jae Won Shin', Ryong Ryoo ${ }^{1,4}$, Yousung Jung (i) ${ }^{2} \&$ Jeong Young Park (i) ${ }^{1,2,4}$

Despite numerous studies, the origin of the enhanced catalytic performance of bimetallic nanoparticles (NPs) remains elusive because of the ever-changing surface structures, compositions, and oxidation states of NPs under reaction conditions. An effective strategy for obtaining critical clues for the phenomenon is real-time quantitative detection of hot electrons induced by a chemical reaction on the catalysts. Here, we investigate hot electrons excited on PtCo bimetallic NPs during $\mathrm{H}_{2}$ oxidation by measuring the chemicurrent on a catalytic nanodiode while changing the Pt composition of the NPs. We reveal that the presence of a $\mathrm{CoO} / \mathrm{Pt}$ interface enables efficient transport of electrons and higher catalytic activity for PtCo NPs. These results are consistent with theoretical calculations suggesting that lower activation energy and higher exothermicity are required for the reaction at the $\mathrm{CoO} / \mathrm{Pt}$ interface.

\footnotetext{
${ }^{1}$ Center for Nanomaterials and Chemical Reactions, Institute for Basic Science (IBS), Daejeon 34141, Republic of Korea. ${ }^{2}$ Graduate School of EEWS, Korea Advanced Institute of Science and Technology (KAIST), Daejeon 34141, Republic of Korea. ${ }^{3}$ School of Energy and Chemical Engineering, Ulsan National Institute of Science and Technology (UNIST), Ulsan 44919, Republic of Korea. ${ }^{4}$ Department of Chemistry, Korea Advanced Institute of Science and Technology (KAIST), Daejeon 34141, Republic of Korea. Correspondence and requests for materials should be addressed to Y.J. (email: ysjn@kaist.ac.kr) or to J.Y.P. (email: jeongypark@kaist.ac.kr)
} 
$\mathrm{n}$ the field of heterogeneous catalysis, there is much interest in understanding how hot electrons, which are associated with energy dissipation and conversion processes during surface reactions, affect catalytic activity and selectivity ${ }^{1-3}$. Extensive experimental studies demonstrate that hot electrons are crucial for explaining the kinetics of catalytic surface reactions because the transport of hot electrons facilitates the formation of a transient state in the molecules ${ }^{4-6}$. However, the microscopic mechanisms of hot-electron-mediated chemistry are still unclear because of the extremely fast relaxation of hot electrons via electron-electron and electron-phonon interactions ${ }^{1}$.

Recently, metal-semiconductor catalytic nanodiodes have been developed as a powerful tool for detecting and utilizing hot electrons generated on nanocatalysts under various surface reactions ${ }^{7-13}$. The architecture of these devices allows for thequick extraction of hot electrons across the metal-semiconductor interface before thermalization, thereby providing key evidence of non-adiabatic charge transfer during surface reactions. In a previous study on $\mathrm{Pt}$ nanoparticle (NP)/Au/TiO 2 catalytic nanodiodes, we showed that the size-dependent catalytic activity of Pt NPs was quantitatively described by the chemicurrent, which is the flow of hot electrons generated on the Pt NPs during a chemical reaction (i.e. in this instance, $\mathrm{H}_{2}$ oxidation) ${ }^{9}$. Furthermore, the kinetics of $\mathrm{H}_{2}$ oxidation following the Langmuir-Hinshelwood mechanism were investigated by measuring the magnitude of the number of hot electrons at different concentrations of hydrogen using $\mathrm{Pt} \quad \mathrm{NP} /$ graphene/TiO 2 nanodiodes ${ }^{10}$.

In contrast with conventional monometallic NPs, bimetallic NPs have opened a new pathway that could control the electronic structure and binding energy in catalysts, resulting in superior catalytic performance ${ }^{4-16}$. Despite considerable focus on various catalytic reaction studies (e.g., catalytic reforming reactions, pollution control, electrochemical catalysis) ${ }^{17,18}$, there are still questions about the underlying causes of improved performance because the structure, chemical composition, and oxidation state of bimetallic NPs can change under reaction conditions ${ }^{19-21}$. Recently, the presence of oxide-metal interfacial sites formed by surface segregation of bimetallic NPs were specifically suggested to be responsible for increased catalytic activity ${ }^{22,23}$. However, the physical nature and fundamental role of oxide-metal interfaces are still elusive because of a lack of definitive evidence.

Herein, we report the real-time detection of hot electrons generated on bimetallic PtCo NPs during exothermic $\mathrm{H}_{2}$ oxidation and clarify the origin of the synergistic catalytic activity of PtCo NPs with corresponding chemicurrent values. To investigate the dynamics of hot electrons on nanocatalysts, we use catalytic $\mathrm{NP} / \mathrm{Au} / \mathrm{TiO}_{2}$ nanodiodes composed of stoichiometric PtCo bimetallic NPs prepared via the co-reduction method using two metals. In both chemicurrent and turnover rate measurements, we observe that the catalytic activity of the bimetallic PtCo NPs is significantly enhanced compared with monometallic Co or Pt NPs. Through X-ray photoelectron spectroscopy (XPS) analysis, transmission electron microscopy (TEM), and densityfunctional theory (DFT) calculations, we confirm that this improvement is attributed to the presence of a $\mathrm{CoO} / \mathrm{Pt}$ interface stabilized on the PtCo NP surface under reaction conditions. By estimating the chemicurrent yield, we conclude that the catalytic properties of the bimetallic NPs are strongly governed by the oxide-metal interface, which facilitates hot electron transfer on the NPs.

\section{Results}

Catalytic nanodiodes with PtCo bimetallic nanoparticles. We present a schematic diagram of a typical device (Fig. 1a) and its energy band diagram (Fig. 1b), where catalytically active NPs (i.e., $\mathrm{Pt}, \mathrm{PtCo}$, Co NPs) were assembled as a two-dimensional (2D) array onto the active surface of $\mathrm{Au} / \mathrm{TiO}_{2}$ nanodiodes using the Langmuir-Blodgett technique. Here, the hot electrons generated on the NPs pass across the $\mathrm{Au} / \mathrm{TiO}_{2}$ Schottky interface if they obtain sufficient energy $(1-3 \mathrm{eV})$ from the chemical reaction to transport irreversibly through the interface. Furthermore, because the thickness of the metal layer is less than the electron mean free path $(<15 \mathrm{~nm})^{9}$, direct detection of the hot electrons is possible before thermalization caused by electron-electron scattering and electron-phonon coupling. To study the dynamics of hot electrons on NPs, monolayer arrays of NPs deposited on $\mathrm{Au} / \mathrm{TiO}_{2}$ nanodiodes were confirmed by scanning electron microscopy (SEM) (Fig. 1c-e and Supplementary Fig. 1). To estimate the conductive properties of the catalytic nanodiodes, current-voltage (I-V) curves were plotted (Fig. 1f). After fitting the I-V curves to the thermionic emission equation, we confirmed that a Schottky barrier was formed at the $\mathrm{Au} / \mathrm{TiO}_{2}$ interface with a height of 0.7 $\mathrm{eV}$ that was preserved regardless of the type of NPs (Fig. 1g; see Supplementary Note 1 for details). This indicates that the Schottky barrier height is not affected by the electronic properties of the NPs deposited on the $\mathrm{Au} / \mathrm{TiO}_{2}$ nanodiode.

Structural and chemical characterization of the $\mathrm{Pt}_{x} \mathrm{Co}_{y} \mathrm{NPs}$. We synthesized $\mathrm{Pt}_{x} \mathrm{Co}_{y}$ NPs with different compositions $(x: y=$ $1: 0,3: 1,1: 1,1: 3,0: 1)$ using the co-reduction method with two precisely mixed metal precursors to study the dynamics of hot electrons on bimetallic NPs. The structures of the $\mathrm{Pt}_{x} \mathrm{Co}_{y} \mathrm{NPs}$ were characterized by TEM, high-angle annular dark-field scanning transmission electron microscopy (HAADF-STEM), and STEM-energy dispersive X-ray spectroscopy (STEM-EDS). As shown in the typical TEM images and size distribution histograms (Fig. 2a-c and Supplementary Fig. 2), the as-synthesized $\mathrm{Pt}_{x} \mathrm{Co}_{y}$ NPs have a uniform size distribution, spherical shape, and high crystallinity. The PtCo bimetallic NPs (i.e., $\mathrm{Pt}_{3} \mathrm{Co}_{1}, \mathrm{Pt}_{1} \mathrm{Co}_{1}$, $\left.\mathrm{Pt}_{1} \mathrm{Co}_{3}\right)$ show an average size range of $2.5-2.8 \mathrm{~nm}$, which are slightly larger than those of the monometallic $\mathrm{Pt}_{1} \mathrm{Co}_{0} \mathrm{NPs}(1.7$ $\mathrm{nm}$ ) and smaller than those of the monometallic $\mathrm{Pt}_{0} \mathrm{Co}_{1} \mathrm{NPs}$ (5.1 $\mathrm{nm})$. The measured $d$-spacings of the PtCo bimetallic NPs are less than the value of the Pt (111) plane (2.26 $\AA$ ). The elemental distributions of the synthesized NPs were assessed using STEM-EDS mapping, which demonstrates that the $\mathrm{Pt}$ and $\mathrm{Co}$ atoms were randomly mixed within the lattice by forming alloys with different stoichiometric ratios of Pt to Co (Supplementary Figs. 2 and 3).

Further chemical analysis of the $\mathrm{Pt}_{x} \mathrm{Co}_{y}$ NPs was carried out using both XPS and inductively coupled plasma mass spectroscopy (ICP-MS) in which the chemical compositions determined by both measurements were well matched with the targeted molar ratios of the Pt and Co precursors (Fig. 2d, Supplementary Fig. 4, and Table 1). By indexing X-ray diffraction (XRD) patterns (Fig. 2e), we examined the microstructure of the $\mathrm{Pt}_{x} \mathrm{Co}_{y} \mathrm{NPs}$ and confirmed that the Pt-rich samples $\left(\mathrm{Pt}_{1} \mathrm{Co}_{0}, \mathrm{Pt}_{3} \mathrm{Co}_{1}\right.$, and $\left.\mathrm{Pt}_{1} \mathrm{Co}_{1}\right)$ exhibit typical (111), (200), and (220) diffraction peaks of the crystalline face-centered cubic (fcc) Pt phase, respectively, with no observable impurities of $\mathrm{CoO}_{x}$ or phase-segregated metals ${ }^{16}$. The peak positions were also shifted slightly to a higher angle as more Co was incorporated into the PtCo NP, thus demonstrating the formation of bimetallic NPs with a disordered alloy phase, which agrees with the STEM-EDS mapping. The lattice parameters calculated from the (220) diffraction peak are 3.925 and $3.851 \AA$ for $\mathrm{Pt}_{1} \mathrm{Co}_{0}$ and $\mathrm{Pt}_{3} \mathrm{Co}_{1}$, respectively, which are consistent with the reported lattice constants of $\mathrm{Pt}$ and $\mathrm{Pt}_{3} \mathrm{Co}_{1}{ }^{24,25}$. However, the pure Co $\left(\mathrm{Pt}_{0} \mathrm{Co}_{1}\right.$, green) NPs show the characteristic pattern indexed to the cubic $\mathrm{Co}_{3} \mathrm{O}_{4}$ structure ${ }^{26}$, indicating that the metallic Co NPs are easily oxidized-even at atmospheric 
a

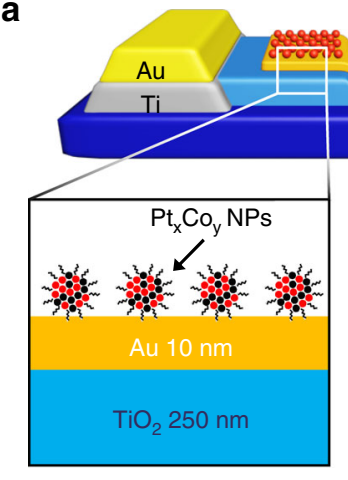

c

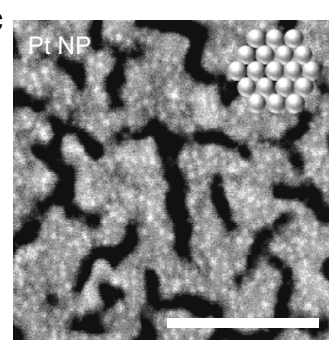

f

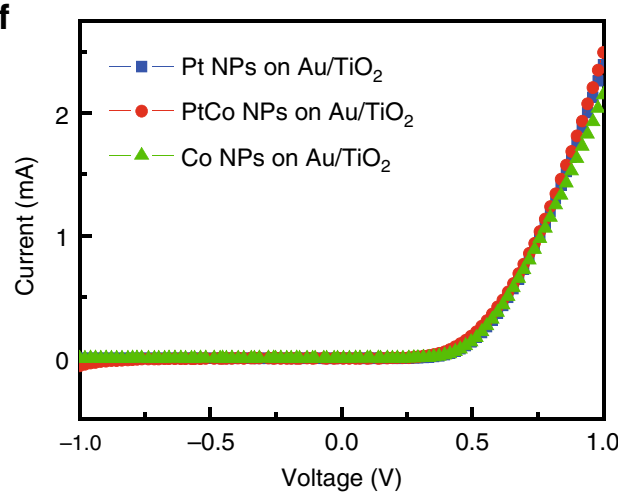

b

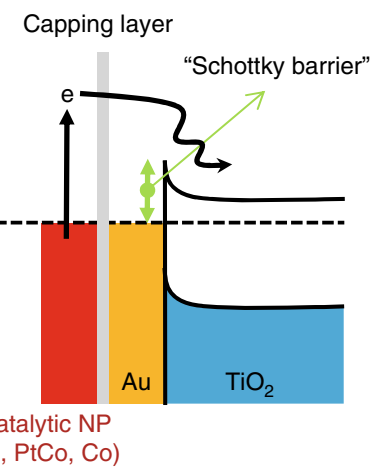

(Pt, PtCo, Co)
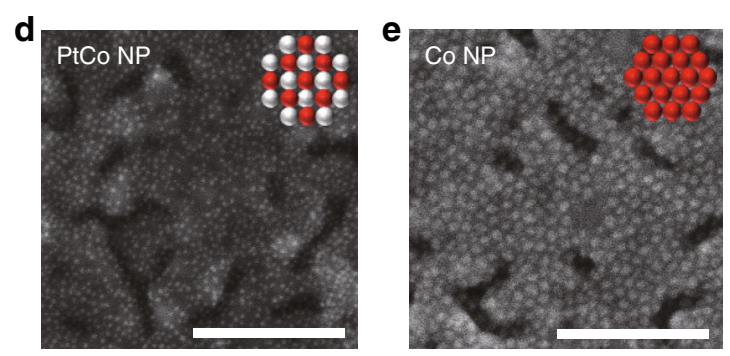

g

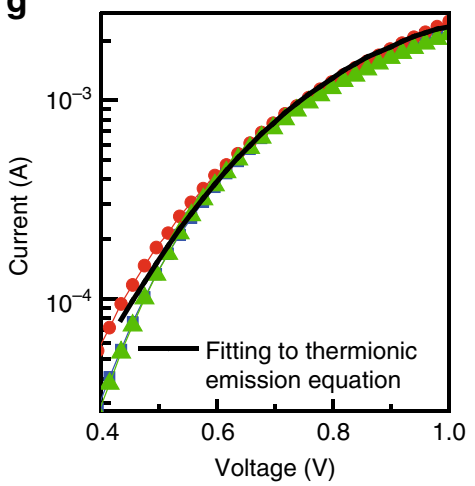

Fig. 1 Detection of hot electrons generated on PtCo bimetallic NPs. a Schematic of a NP/Au/ $\mathrm{TiO}_{2}$ catalytic nanodiode and cross-sectional field emission scanning electron microscopy (FE-SEM) image of a $10 \mathrm{~nm}$ Au film on a $250 \mathrm{~nm} \mathrm{TiO}$ layer. The rectifying contact is formed at the interface between the Au and $\mathrm{TiO}_{2}$ layers. The Ti layer makes the ohmic junction with the $\mathrm{TiO}_{2}$. Scale bar in FE-SEM image is $300 \mathrm{~nm}$. b Energy band diagram for the Au/TiO nanodiode with various bimetallic NPs with different compositions. Hot electrons energetic enough to overcome the Schottky barrier can be detected as a steady-state current. Scanning electron microscopy (SEM) images of a monolayer of $\mathbf{c}$ Pt, d PtCo, and e Co NPs on a $10 \mathrm{~nm}$ Au layer. Scale bars are $100 \mathrm{~nm}$ (c-e). f Current-voltage (I-V) curves measured on the $\mathrm{Au} / \mathrm{TiO}_{2}$ catalytic nanodiodes with Pt (blue), PtCo (red), and Co (green) NPs. $\mathbf{g}$ Fitting the I-V curves of the $\mathrm{Au} / \mathrm{TiO}_{2}$ nanodiode to the thermionic emission equation. The catalytic nanodiodes show a Schottky barrier height of $0.7 \mathrm{eV}$

conditions-to $\mathrm{Co}_{3} \mathrm{O}_{4}$, which is thermodynamically more stable than the $\mathrm{CoO}$ phase. The average crystallite sizes were calculated from XRD using the Debye-Scherrer equation; they are consistent with the NP sizes obtained from TEM (Supplementary Table 2). Overall analyses confirm that the resulting $\mathrm{Pt}_{x} \mathrm{Co}_{y} \mathrm{NPs}$ have uniform size and well-controlled stoichiometric $\mathrm{Pt} / \mathrm{Co}$ ratios.

Synergistic catalytic activity of the PtCo NPs. As a model system, the catalytic oxidation of hydrogen $\left(\mathrm{H}_{2}+\mathrm{O}_{2} \rightarrow \mathrm{H}_{2} \mathrm{O}\right)$ has been chosen for the reaction kinetic study because it is a simple and representative reaction and it is closely correlated to a variety of hydrogen-based energy systems. To investigate the flow of hot electrons induced by the exothermic reaction on the catalytic $\mathrm{NPs}$, we carried out current measurements on the $\mathrm{Pt}_{x} \mathrm{Co}_{y} \mathrm{NP} / \mathrm{Au} /$ $\mathrm{TiO}_{2}$ catalytic nanodiodes in a $\mathrm{H}_{2}$ (15 Torr) $+\mathrm{O}_{2}$ (745 Torr) mixture as well as in pure $\mathrm{O}_{2}$ at elevated temperatures (i.e., $\left.30-120^{\circ} \mathrm{C}\right)$. We observed a definite deviation between the currents measured with and without catalytic $\mathrm{H}_{2}$ oxidation, indicating that the differences in magnitude of the currents were associated with hot electrons (i.e., chemicurrent) generated on the surface of the $\mathrm{Pt}_{x} \mathrm{Co}_{y}$ NPs (Fig. 3a; see Supplementary Note 2 and Fig. 5 for details). The chemical nature of the observed currents was confirmed using a $30 \mathrm{~nm}$ Au layer where the chemicurrent drops to zero due to attenuation of the hot electrons?

Figure $3 \mathrm{~b}$ shows the overall chemicurrent for the $\mathrm{Pt}_{x} \mathrm{Co}_{y} \mathrm{NPs}$; the current signals changed as a function of Co content and the $\mathrm{Pt}_{3} \mathrm{Co}_{1}$ bimetallic NPs exhibit the highest values during the catalytic $\mathrm{H}_{2}$ oxidation reaction over the entire temperature range. In addition, it is noteworthy that the chemicurrents of the large NPs, including $\mathrm{Pt}_{3} \mathrm{Co}_{1}, \mathrm{Pt}_{1} \mathrm{Co}_{1}$, and $\mathrm{Pt}_{1} \mathrm{Co}_{3}(2.5 \mathrm{~nm})$, exhibit a somewhat higher current than the smaller $\mathrm{Pt}_{1} \mathrm{Co}_{0} \mathrm{NPs}(1.7 \mathrm{~nm})$, even though the detection efficiency of hot electrons is higher in smaller NPs due to the shorter travel length for the hot electrons ${ }^{9}$. This implies that the enhanced chemicurrent obtained on the PtCo bimetallic NPs indeed originates from their improved catalytic properties and is beyond the size effect of the NPs. 

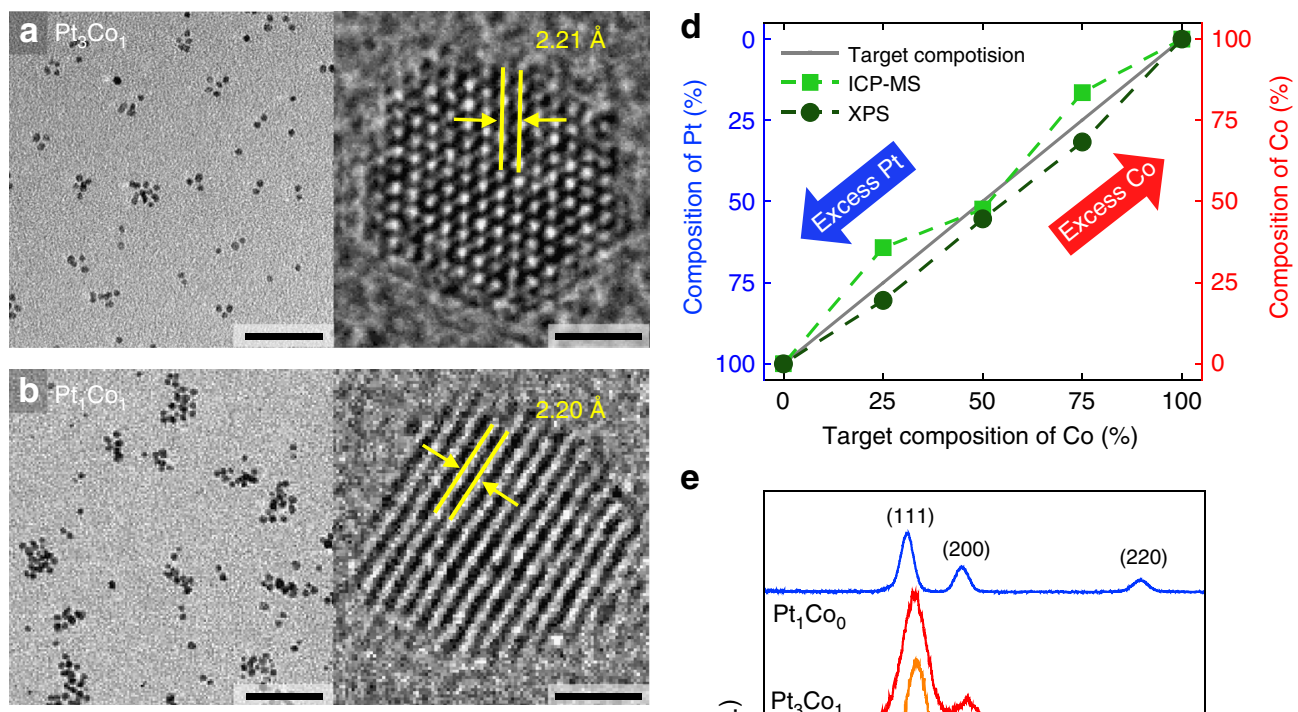

e

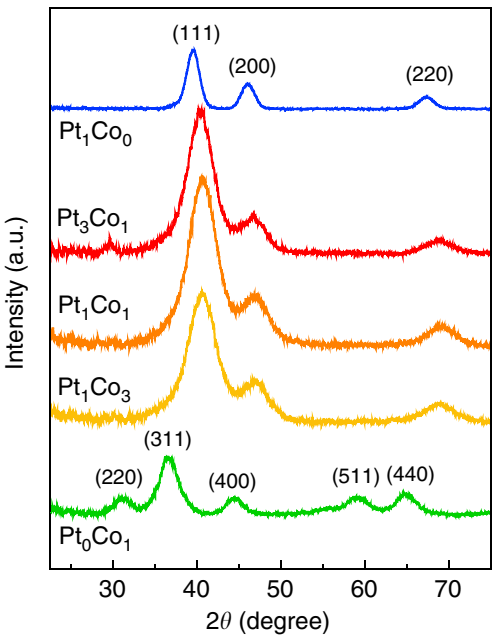

Fig. 2 Structural and chemical characterization of PtCo bimetallic NPs. TEM and high-resolution TEM (HRTEM) images of as-synthesized $\mathbf{a} \mathrm{Pt}_{3} \mathrm{Co}_{1}$, $\mathbf{b}$ $\mathrm{Pt}_{1} \mathrm{Co}_{1}$, and $\mathbf{c} \mathrm{Pt}_{1} \mathrm{Co}_{3}$ bimetallic NPs. Scale bars are $30 \mathrm{~nm}$ (left) and $1 \mathrm{~nm}$ (right) (a-c). d Comparison of the composition values of the Pt $\mathrm{Co}_{y}$ bimetallic NPs obtained from XPS and ICP-MS, which generally agree well with the targeted molar ratio of the $\mathrm{Co}$ and $\mathrm{Pt}$ precursors. e XRD patterns of $\mathrm{Pt}_{1} \mathrm{Co}_{0}$ (blue), $\mathrm{Pt}_{3} \mathrm{Co}_{1}$ (red), $\mathrm{Pt}_{1} \mathrm{Co}_{1}$ (orange), $\mathrm{Pt}_{1} \mathrm{Co}_{3}$ (yellow), and $\mathrm{Pt}_{0} \mathrm{Co}_{1}$ (green) NPs

Concurrently, the observed trend of the chemicurrent agrees well with the turnover frequency (TOF) measured on the monolayered $\mathrm{Pt}_{x} \mathrm{Co}_{y} \mathrm{NPs}$ supported on $\mathrm{SiO}_{2}$ substrates at temperatures of $80-110^{\circ} \mathrm{C}$ under identical reaction conditions (Fig. 3c). Here, to ensure the reversibility of the chemicurrent measurement, every measurement was repeated 2-3 times for all experiments and it was confirmed that the effect of the substrate on the catalytic activity is insignificant (Supplementary Figs. 7 and 8). The catalytic activities of the PtCo bimetallic NPs are significantly enhanced, while the monometallic $\mathrm{Pt}_{1} \mathrm{Co}_{0}$ or $\mathrm{Pt}_{0} \mathrm{Co}_{1} \mathrm{NPs}$ show only moderate or no activity for the $\mathrm{H}_{2}$ oxidation reaction, respectively. Moreover, as the Co content increased in the PtCo bimetallic NPs, both the chemicurrent and TOF values decreased and approached the signal of the $\mathrm{Pt}_{1} \mathrm{Co}_{0}$ NPs (Fig. $3 \mathrm{~d}$ and Supplementary Fig. 9). These findings suggest that Co alloying with $\mathrm{Pt}$ at nanoscale has a synergistic effect on the catalytic reaction, and that the magnitude of the number of hot electrons captured by the catalytic nanodiodes is an instrumental descriptor of the catalytic properties regardless of the type of catalyst.

Origin of the synergistic activity in the PtCo NPs. Because of the complexity of the system, the origin of the synergistic catalytic effect found in bimetallic NPs is still debated. One of the most plausible reasons for the enhanced catalytic performance is the presence of oxide-metal interfacial sites, which are formed at the surface of the NPs during the catalytic reaction. To confirm the presence of $\mathrm{CoO} / \mathrm{Pt}$ interfacial sites on the surface of our PtCo bimetallic $\mathrm{NPs}$ (i.e., $\mathrm{Pt}_{3} \mathrm{Co}_{1}, \mathrm{Pt}_{1} \mathrm{Co}_{1}, \mathrm{Pt}_{1} \mathrm{Co}_{3}$ ), we conducted ex situ
XPS and in situ TEM measurements. As shown in Fig. 4a, the chemical states of the cobalt in the $\mathrm{Pt}_{3} \mathrm{Co}_{1}$ NPs consist of metallic $\mathrm{Co}$ and $\mathrm{CoO}$ and the amount of $\mathrm{CoO}$ species increased by $23 \%$ because of further oxidation during $\mathrm{H}_{2}$ oxidation. Unlike cobalt, regardless of the composition of the PtCo bimetallic NPs, platinum is rarely oxidized and maintains its metallic properties during the reaction (Supplementary Note 3 and Supplementary Figs. 10-14). Therefore, in the PtCo bimetallic NPs, it is definitive that the cobalt was easily oxidized and segregated on the metallic Pt surface as a form of $\mathrm{CoO}$ under the $\mathrm{H}_{2}$ oxidation conditions ${ }^{27,28}$. We also noticed that the amount of $\mathrm{CoO}$ existing on the surface of the PtCo NPs increased as the $\mathrm{Co} / \mathrm{Pt}$ ratio increased, resulting in a reduced interfacial area of the $\mathrm{CoO} / \mathrm{Pt}$ on the NPs (Fig. 4b; see Supplementary Note 3 for more details).

Furthermore, to determine the structural configuration of the $\mathrm{CoO}$ layer formed in the PtCo NPs, in situ TEM experiments were performed using an aberration-corrected environmental TEM (Titan ETEM G2, FEI) with a Fusion heating holder (Protochips inc.), enabling dynamic observation of the NP surface at atomic-scale resolution (Supplementary Note 4). For the in situ TEM measurements, $\mathrm{Pt}_{3} \mathrm{Co}_{1}$ NPs were supported on spherical silica particles with diameters of $380 \mathrm{~nm}$ and the $\mathrm{Pt}_{3} \mathrm{Co}_{1} / \mathrm{SiO}_{2}$ sample was heated from room temperature to $125^{\circ} \mathrm{C}$ at a heating rate of $25^{\circ} \mathrm{C} \mathrm{min}^{-1}$ in $0.5 \mathrm{mbar}$ of $\mathrm{O}_{2}$ gas inside the TEM. While monitoring the change in the surface structure of the $\mathrm{Pt}_{3} \mathrm{Co}_{1} \mathrm{NPs}$ under oxidation conditions $\left(0.5 \mathrm{mbar} \mathrm{O}_{2}\right.$ at $\left.125^{\circ} \mathrm{C}\right)$, we found that additional Co atoms began to segregate after a few seconds ( $14 \mathrm{~s}$ ), grew atom-by-atom along the $\{111\}$ surface of the $\mathrm{Pt}_{3} \mathrm{Co}_{1}$ $\mathrm{NP}(\sim 54 \mathrm{~s})$, and formed a monolayer of $\mathrm{CoO}\{111\}$, which is 
a

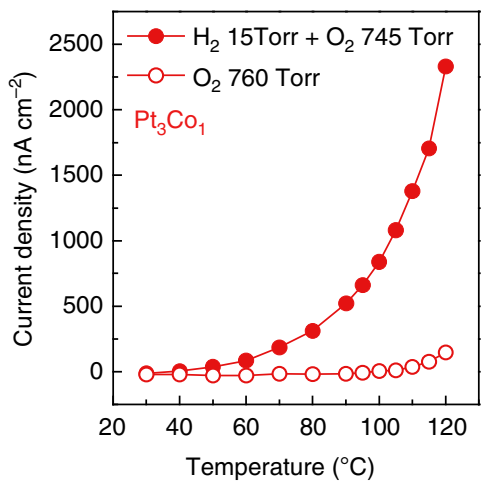

b



C

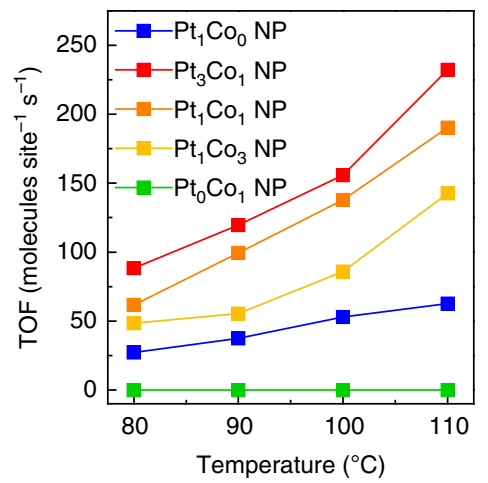

d

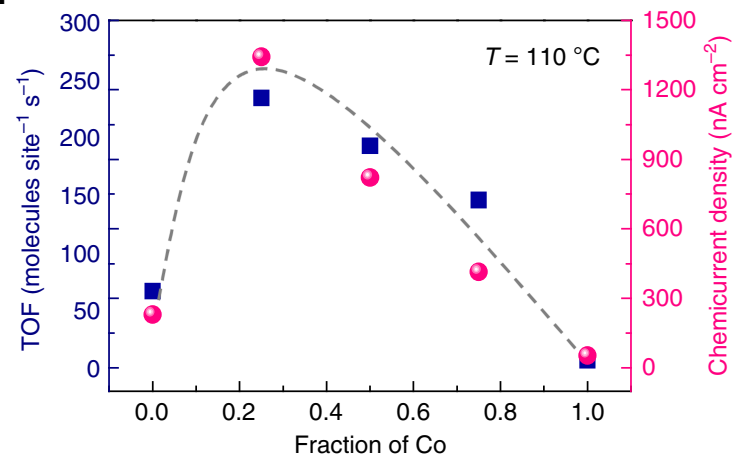

e

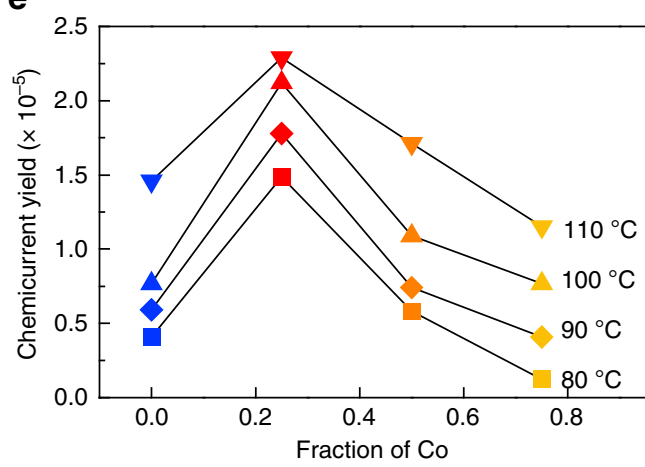

Fig. 3 Hot electron detection and catalytic activity on PtCo bimetallic NPs. a Temperature dependence of the current from the $\mathrm{Au} / \mathrm{TiO}_{2}$ nanodiodes with $\mathrm{Pt}_{3} \mathrm{Co}_{1}$ NPs measured in the $\mathrm{H}_{2}+\mathrm{O}_{2}$ gas mixture and in pure $\mathrm{O}_{2}$. b Chemicurrents associated with the $\mathrm{H}_{2}$ oxidation reaction measured on the Au/TiO $\mathrm{O}_{2}$ nanodiodes at different temperatures with $\mathrm{Pt}_{x} \mathrm{Co}_{y}$ NPs of different compositions; the data were normalized based on the total NP surface area. c Catalytic activity (i.e., TOF) for $\mathrm{H}_{2}$ oxidation on a set of $\mathrm{Pt}_{x} \mathrm{Co}_{y}$ NPs with different compositions. $\mathbf{d}$ Comparison of both the chemicurrent and TOF as a function of the composition of the $\mathrm{PtC}$ bimetallic NPs at $110^{\circ} \mathrm{C}$. e Plot of the chemicurrent yield as a function of the fraction of Co during $\mathrm{H}_{2}$ oxidation at different temperatures

a



b

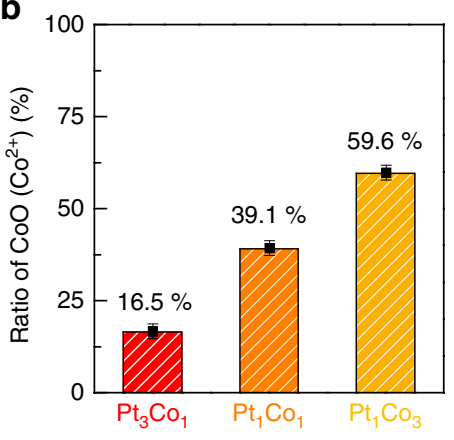

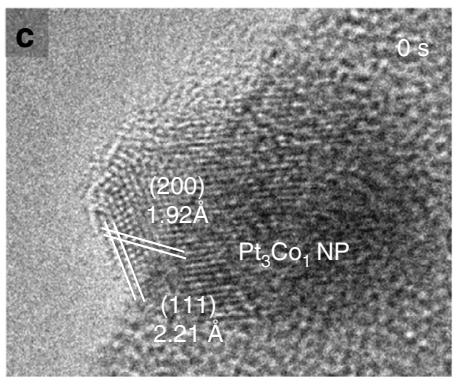
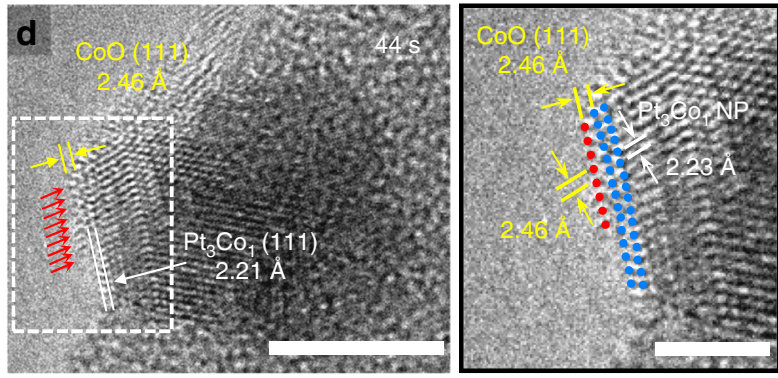

Fig. 4 Formation of $\mathrm{CoO}$ on PtCo bimetallic NPs. a Co $2 p$ XPS spectra showing the oxidation states of the Co in the $\mathrm{Pt}_{x} \mathrm{Co}_{y}$ bimetallic NPs before and after $\mathrm{H}_{2}$ oxidation. b Relative ratio of $\mathrm{CoO}$ in the $\mathrm{Pt}_{x} \mathrm{Co}_{y}$ bimetallic NPs estimated using the peak area of $\mathrm{Co}^{2+}$. Error bars are $\pm s d$. Sequential in situ TEM images of the $\mathrm{Pt}_{3} \mathrm{Co}_{1} \mathrm{NP}$ taken at $\mathbf{c} 0$ and $\mathbf{d} 36 \mathrm{~s}$ during oxidization at $125^{\circ} \mathrm{C}$ in $0.5 \mathrm{mbar}$ of $\mathrm{O}_{2}$ gas. As denoted by the red arrows, additional Co atoms segregated on the $\mathrm{Pt}_{3} \mathrm{Co}_{1} \mathrm{NP}$ surface. The difference in lattice spacing between the top-most layer and the inner portion is clearly shown in the enlargement region denoted by the white rectangle. Scale bars are $5 \mathrm{~nm}$ (left) and $2 \mathrm{~nm}$ (right) 
identified by lattice spacing measurements (Fig. 4c, d, Supplementary Fig. 15 and Movie 1). The red arrows on the top surface in Fig. $4 \mathrm{~d}$ indicate Co atoms segregated on the NP surface. The lattice spacing between the outmost surface and the subsurface layer is $2.46 \AA$, which is larger than the $2.21 \AA$ of $\mathrm{Pt}_{3} \mathrm{Co}_{1}\{111\}$ and identical to the d-spacing of $\mathrm{CoO}\{111\}$ (Fig. 4d). It was also confirmed that the CoO formed on the top-most layer of the NP was maintained even after 15 min (Supplementary Fig. 16). The formation and stabilization of the CoO layer on the PtCo NPs under oxidizing conditions is plausible because of the dominant interfacial strain at the $\mathrm{CoO} / \mathrm{Pt}$ caused from lattice mismatch, or the strong interaction (i.e., so-called interface confinement effect) of $\mathrm{Co}-\mathrm{Pt}$ binding at the $\mathrm{CoO} / \mathrm{Pt}$ surface ${ }^{29-31}$. Therefore, the much-improved catalytic activity of the PtCo bimetallic NPs could be ascribed to the presence of $\mathrm{CoO} / \mathrm{Pt}$ interfacial sites on the catalyst surface; and consequently, increasing the interfacial region between the Co and Pt on the PtCo NPs leads to a higher catalytic activity for $\mathrm{H}_{2}$ oxidation.

However, the unique electronic properties of the oxide-metal interface are still somewhat ambiguous, especially at nanoscale, due to a lack of concrete experimental data ${ }^{32}$. To overcome this challenge, we estimated the chemicurrent yield, which is the detection probability of hot electrons when one molecule of product is formed during the surface reaction (Fig. 3e). Because the magnitude of the chemicurrent is proportional to the reaction rate, the chemicurrent can be expressed as $I_{\mathrm{ch}}=\alpha q A N \cdot$ TOF, where $\alpha$ is the chemicurrent yield, $q$ is the elementary charge, $A$ is the active area of the catalyst, and $N$ is the number of metal sites per square millimeter ${ }^{1}$. Here, the chemicurrent yield is determined by the distribution of hot electrons, attenuation in the metal, and the transmission probability across the Schottky barrier. Therefore, if both the NP size and Schottky barrier height of the nanodiodes remain the same, the chemicurrent yields are consistent at a given temperature. However, as shown in Fig. 3e, the chemicurrent yield varies depending on the composition of the PtCo NPs at a constant temperature, and the highest value is measured on the $\mathrm{Pt}_{3} \mathrm{Co}_{1} \mathrm{NPs}$ with the largest interfacial area of $\mathrm{CoO} / \mathrm{Pt}$.

This intriguing result could be attributed to local polarization at the $\mathrm{CoO} / \mathrm{Pt}$ interface that originates from charge transfer between the metal and the oxide, which contributes to an acceleration of hot electron transport and to prolonging the lifetime of the hot electrons at the interfacial sites ${ }^{32,33}$. Therefore, the decrease in chemicurrent yield with increased $\mathrm{CoO}$ coverage indicates that the interfacial $\mathrm{CoO} / \mathrm{Pt}$ region is reduced, resulting in less-efficient electron transfer on the PtCo NPs. These are important findings because they are the first visualization of the unique electronic structure of the oxide-metal interface in bimetallic NPs during a chemical reaction obtained through direct measurement of hot electrons.

\section{Discussion}

To gain insight into the enhanced catalytic activity of the interfacial sites between the $\mathrm{CoO}$ and $\mathrm{Pt}$, we investigated the reaction energetics of the $\mathrm{H}_{2}$ oxidation reaction using density-functional theory (DFT) calculations ${ }^{34}$. For our model system, the $\mathrm{CoO}$ island structure on a Pt (111) surface, denoted as $\mathrm{CoO} / \mathrm{Pt}$, was investigated and compared with the Pt (111) surface (Supplementary Fig. 17$)^{29,35}$. ${ }^{\star} \mathrm{OH}$ formation is calculated to be the ratedetermining step in the $\mathrm{H}_{2}$ oxidation reaction for both the $\mathrm{Pt}$ (111) and $\mathrm{CoO} / \mathrm{Pt}$ interface sites (Fig. 5a, b) ${ }^{36-38}$. However, the activation barrier for ${ }^{\star} \mathrm{OH}$ formation at the $\mathrm{CoO} / \mathrm{Pt}$ interface is smaller than that for $\operatorname{Pt}(111)$ by $0.16 \mathrm{eV}$. This result can be understood by the $\mathrm{Pt}-\mathrm{O}$ bond at the $\mathrm{CoO} / \mathrm{Pt}$ interface not undergoing major atomic rearrangement when forming ${ }^{\star} \mathrm{OH}$, while the same $\mathrm{Pt}-\mathrm{O}$ bond on the $\mathrm{Pt}$ (111) surface requires the transfer of ${ }^{\star} \mathrm{O}$ from the hollow site to the top site to form ${ }^{\star} \mathrm{OH}$ (Supplementary Fig. 18 and Table 3). In the subsequent reaction steps on the $\mathrm{CoO} / \mathrm{Pt}$ interface, ${ }^{\star} \mathrm{H}$ migrates from the $\mathrm{CoO}$ to the nearby ${ }^{\star} \mathrm{O}$ site on the $\mathrm{Pt}$, followed by ${ }^{\star} \mathrm{H}-{ }^{\star} \mathrm{OH}$ coupling to release $\mathrm{H}_{2} \mathrm{O}$ (Fig. 5 c; see Supplementary Note 5 for more details). We also confirmed that the energy released during $\mathrm{H}_{2}$ oxidation at the $\mathrm{CoO} / \mathrm{Pt}$ interface $(1.17 \mathrm{eV})$ is higher than that on the $\mathrm{Pt}(111)$ surface $(0.79 \mathrm{eV})$, supporting the experimental results that hot electron generation is enhanced on the PtCo bimetallic NPs with a $\mathrm{CoO} / \mathrm{Pt}$ interface. All these results thus point to the conclusion that local electronic perturbations caused by the presence of the $\mathrm{CoO} / \mathrm{Pt}$ interface do indeed contribute to enhanced catalytic activity of the PtCo bimetallic NPs. Since it is possible that the reaction could also occur on the $\mathrm{CoO}$ cluster (island) itself instead a

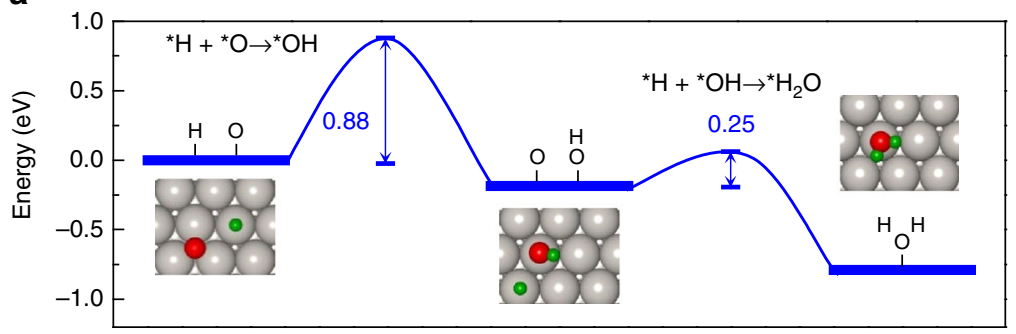

$\mathrm{Pt}$

b

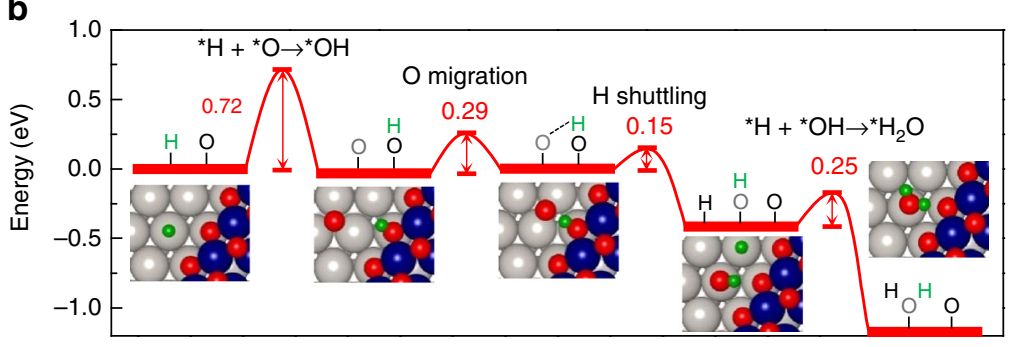

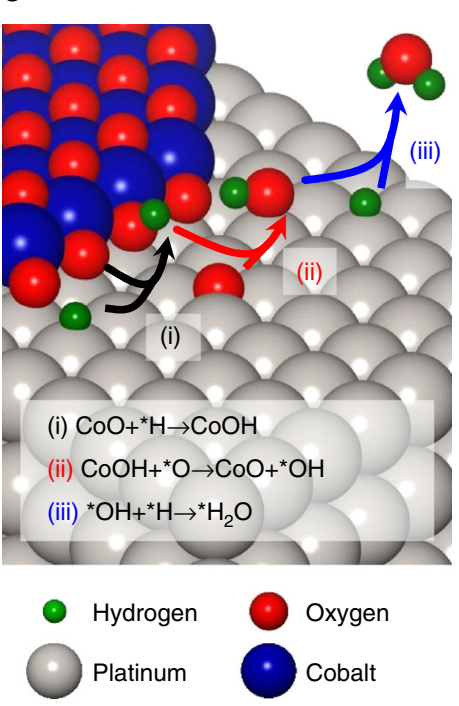

$\mathrm{CoO} / \mathrm{Pt}$

Fig. 5 Reaction pathways of $\mathrm{H}_{2}$ oxidation calculated using DFT. Schematic energy diagram of the $\mathrm{H}_{2}$ oxidation reaction $\mathbf{a}$ on the $\mathrm{Pt}$ (111) surface and $\mathbf{b}$ at the $\mathrm{CoO} / \mathrm{Pt}$ interface. c Drawing showing the $\mathrm{H}_{2}$ oxidation reaction mechanism at the periphery of the CoO/Pt interface 
of at the $\mathrm{CoO} / \mathrm{Pt}$ interface, we also considered the same reactions occurring at the top sites of the island model of the cobalt monoxide. The high activation barrier $(1.34 \mathrm{eV})$ indicates that $\mathrm{H}_{2}$ oxidation on the cobalt monoxide cluster itself will be unlikely (Supplementary Fig. 20).

In this study, we have quantitatively correlated the catalytic activity of PtCo NPs with the magnitude of hot electron flow generated during $\mathrm{H}_{2}$ oxidation via real-time chemicurrent measurements using a metal-semiconductor catalytic nanodiode. Based on both the chemicurrent and TOF results, we conclude that the synergistic catalytic performance of the PtCo NPs originates from the presence of the $\mathrm{CoO} / \mathrm{Pt}$ interface under reaction conditions. Using DFT calculations, we also confirmed that the reduced energy barrier for $\mathrm{OH}$ formation at the $\mathrm{CoO} / \mathrm{Pt}$ interface promoted the overall catalytic $\mathrm{H}_{2}$ oxidation reaction. Most importantly, the composition-dependent chemicurrent yield proved that the locally modified electronic structure at the metal-oxide interface played a decisive role in improving the catalytic activity of the PtCo NPs. The present measurements are the first visualization of the unique electronic structure of the oxide-metal interface in bimetallic NPs during a chemical reaction, which were obtained through direct detection of hot electrons. This provides a clear message that chemicurrent experiments are a reliable way to investigate hot electron dynamics on nanocatalysts during chemical reactions.

\section{Methods}

Synthesis of the $\mathbf{P t}_{\boldsymbol{x}} \mathbf{C o}_{\boldsymbol{y}}$ NPs. To prepare the PtCo bimetallic NPs with wellcontrolled stoichiometry, we used the conventional polyol reduction method in which co-reduction of two metal precursors occurred and the initial concentration ratio of the cobalt-to-platinum precursors determined the final stoichiometry. Further details of the synthesis are described in the Supplementary Methods.

Fabrication of the catalytic nanodiode. The metal-semiconductor nanodiodes were fabricated as follows: First, we deposited a thin $(250 \mathrm{~nm})$ layer of Ti on a 500 $\mathrm{nm} \mathrm{SiO}_{2}$ layer/p-type $\mathrm{Si}(100)$ using electron beam evaporation with a titanium target and a patterned aluminum shadow mask $\left(4 \times 6 \mathrm{~mm}^{2}\right)$. To modify the Fermi level of the $\mathrm{TiO}_{2}$ film, the wafer was annealed in air at $380{ }^{\circ} \mathrm{C}$ for $2 \mathrm{~h}$, while the sheet resistance was monitored. The electrode, a $50 \mathrm{~nm}$ Ti layer and $150 \mathrm{~nm} \mathrm{Au}$ layer, was then deposited using electron beam evaporation through a second aluminum shadow mask $\left(5 \times 5 \mathrm{~mm}^{2}\right)$. The titanium layer is the ohmic contact with the $\mathrm{TiO}_{2}$ thin film and the Au layer comprises the nanodiode's two ohmic electrodes. Finally, a 10 or $30 \mathrm{~nm}$ Au thin film was deposited through a third patterned mask $\left(2 \times 1 \mathrm{~mm}^{2}\right)$. This produced $\mathrm{Au} / \mathrm{TiO}_{2}$ nanodiodes with a $4 \mathrm{~mm}^{2}$ active area.

To assemble the 2D monolayer of $\mathrm{Pt}_{x} \mathrm{Co}_{y}$ NPs $(x: y=1: 0,3: 1,1: 1,1: 3,0: 1)$ on the $\mathrm{Au} / \mathrm{TiO}_{2}$ nanodiode, we used the Langmuir-Blodgett technique. The solution of $\mathrm{Pt}_{x} \mathrm{Co}_{y}$ NPs was first dispersed in water on a Langmuir-Blodgett trough (Nima Technology, M611) at room temperature. As the water-supported thin film layer of nanoparticles reached equilibrium, the layer was compressed by moving the mobile barrier at a rate of $15 \mathrm{~cm}^{2} \mathrm{~min}^{-1}$ while monitoring the surface pressure with a Wilhelmy plate. Finally, 2D arrays of $\mathrm{Pt}_{x} \mathrm{Co}_{y} \mathrm{NPs}$ on the $\mathrm{Au} / \mathrm{TiO}_{2}$ nanodiode were created by lifting the submerged substrate from the water. Before setting the diode into the water, the nonactive portion of the diode surface was covered with Teflon tape, and the $\mathrm{Pt}_{x} \mathrm{Co}_{y}$ NPs were left only on the active diode area (i.e., the thin $10 \mathrm{~nm}$ Au layer in contact with the $\mathrm{TiO}_{2}$ ) after removing the Teflon tape. The resulting monolayer arrays of NPs on the $\mathrm{Au} / \mathrm{TiO}_{2}$ nanodiode and $\mathrm{SiO}_{2}$ substrate were confirmed by SEM, as shown in Fig. 1 and Supplementary Fig. 1, respectively.

TOF measurement. The $\mathrm{H}_{2}$ oxidation reaction was performed in an ultra-high vacuum batch reactor $(1 \mathrm{~L})$ with a base pressure of $5.0 \times 10^{-8}$ Torr. The reaction chamber was evacuated and isolated with a gate valve before it was charged with 15 Torr of $\mathrm{H}_{2}$ and 745 Torr of $\mathrm{O}_{2}$ at room temperature. A catalyst sample was placed on a ceramic heater in the batch reactor, and the temperature was monitored by a thermocouple and fluctuated no more than $0.5 \mathrm{~K}$. The reaction mixture was circulated continuously through the reaction line by a Metal Bellows recirculation pump at a rate of $2 \mathrm{~L} \mathrm{~min}^{-1}$. The $\mathrm{H}_{2}$ molecules were monitored as a function of reaction temperature $\left(80-110^{\circ} \mathrm{C}\right)$. After equilibrating for $1 \mathrm{~h}$, the reaction mixture was continuously analyzed through an online gas chromatograph. The reaction mixture was separated for analysis using a DS iGC 7200 gas chromatograph equipped with a thermal conductivity detector and a $6 \mathrm{ft}$ long, $1 / 8^{\prime \prime}$ outer diameter stainless steel 80/100 mesh size column. $\mathrm{H}_{2} \mathrm{O}$ conversion was reported in terms of TOF and was calculated on the basis of product molecules of $\mathrm{H}_{2} \mathrm{O}$ produced per metal surface site per second of reaction time.
Characterization. XPS spectra were acquired using a Thermo VG Scientific Sigma Probe spectrometer equipped with an Al-Ka X-ray source $(1486.3 \mathrm{eV})$ and an energy resolution of $0.5 \mathrm{eV}$ full width at half maximum under ultra-high vacuum conditions of $10^{-10}$ Torr. The TEM measurements were performed using a Tecnai TF30 ST at $300 \mathrm{kV}$ and HRTEM, HAADF-STEM, and STEM-EDS mapping analyses were performed using a Titan cubed G2 60-300 (FEI) at $300 \mathrm{kV}$ with a spherical aberration corrector (CEOS GmbH). EDS analysis was carried out along with four integrated silicon-drift EDS detectors (ChemiSTEM ${ }^{\mathrm{TM}}$ technology) at a collection solid angle of $0.7 \mathrm{srad}$. An aberration-corrected environmental TEM (Titan ETEM G2, FEI) operating at $300 \mathrm{kV}$ with the Fusion heating holder (Protochips inc.) was used for the in situ TEM characterization. Field emission SEM was conducted using a Verios 460 SEM instrument. For electrical characterization of the nanodiodes, I-V curves and the short-circuit current were measured using a Keithley Instrumentation 2400 sourcemeter under various chemical reaction conditions. The reaction rates of the $\mathrm{H}_{2}$ oxidation reaction were measured using a gas chromatograph (DS iGC 7200) in a batch reactor system.

Simulation methods. Spin-polarized density-functional theory calculations were performed to optimize the atomic structures and calculate the electronic energies using the Vienna Ab initio simulation package code $\mathrm{c}^{39-42}$. The D3-corrected ${ }^{43}$ revised Perdew-Burke-Ernzerhof exchange-correlation functional ${ }^{44,45}$ with the projector augmented wave method was used ${ }^{39,46}$, and the kinetic cutoff energy was set to $500 \mathrm{eV}$. The nudged elastic band method was employed to find a minimum energy pathway and to calculate the activation barriers ${ }^{47,48}$. Geometry optimization and nudged elastic band calculations were performed until the residual force on each atom was less than $0.05 \mathrm{eV} \AA^{-1}$ and $0.1 \mathrm{eV} \AA^{-1}$, respectively. K-points were sampled using a $2 \times 2 \times 1$ Monkhorst-Pack mesh ${ }^{49}$. To represent the Pt (111) surface, 48 atoms in a $(4 \times 4)$ surface unit cell with three atomic layers was modeled with spacing more than $15 \AA$ in the z-direction. To represent the CoO/Pt interface, we modeled periodic $\mathrm{CoO}$ islands on a three-layered Pt (111) slab (Supplementary Fig. 17) 29,35 .

Data availability. The authors declare that all data supporting the findings of this study are available within the paper and its supplementary information files.

Received: 11 November 2017 Accepted: 17 May 2018

Published online: 08 June 2018

\section{References}

1. Park, J. Y., Kim, S. M., Lee, H. \& Nedrygailov, I. I. Hot-electron-mediated surface chemistry: Toward electronic control of catalytic activity. Acc. Chem. Res. 48, 2475-2483 (2015)

2. Wodtke, A. M. Electronically non-adiabatic influences in surface chemistry and dynamics. Chem. Soc. Rev. 45, 3641-3657 (2016)

3. Brongersma, M. L., Halas, N. J. \& Nordlander, P. Plasmon-induced hot carrier science and technology. Nat. Nanotechnol. 10, 25-34 (2015).

4. Bonn, M. et al. Phonon-versus electron-mediated desorption and oxidation of CO on Ru (0001). Science 285, 1042-1045 (1999).

5. Bünermann, O. et al. Electron-hole pair excitation determines the mechanism of hydrogen atom adsorption. Science 350, 1346-1349 (2015).

6. Zhou, L. et al. Aluminum nanocrystals as a plasmonic photocatalyst for hydrogen dissociation. Nano Lett. 16, 1478-1484 (2016).

7. Gergen, B., Nienhaus, H., Weinberg, W. H. \& McFarland, E. W. Chemically induced electronic excitations at metal surfaces. Science 294, 2521-2523 (2001).

8. Karpov, E. \& Nedrygailov, I. Solid-state electric generator based on chemically induced internal electron emission in metal-semiconductor heterojunction nanostructures. Appl. Phys. Lett. 94, 214101 (2009).

9. Lee, H., Nedrygailov, I. I., Lee, C., Somorjai, G. A. \& Park, J. Y. Chemical reaction-induced hot electron flows on Pt colloid nanoparticles under hydrogen oxidation: Impact of nanoparticle size. Angew. Chem. Int. Ed. 54, 2340-2344 (2015)

10. Lee, H. et al. Graphene-semiconductor catalytic nanodiodes for quantitative detection of hot electrons induced by a chemical reaction. Nano Lett. 16, 1650-1656 (2016)

11. Nedrygailov, I. I., Lee, C., Moon, S. Y., Lee, H. \& Park, J. Y. Hot electrons at solid-liquid interfaces: A large chemoelectric effect during the catalytic decomposition of hydrogen peroxide. Angew. Chem. Int. Ed. 128, 11017-11020 (2016)

12. Nienhaus, H. Electronic excitations by chemical reactions on metal surfaces. Surf. Sci. Rep. 45, 1-78 (2002).

13. Park, J. Y. \& Somorjai, G. A. The catalytic nanodiode: Detecting continous electron flow at oxide-metal interfaces generated by a gas-phase exothermic reaction. Chemphyschem 7, 1409-1413 (2006). 
14. Gilroy, K. D., Ruditskiy, A., Peng, H. -C., Qin, D. \& Xia, Y. Bimetallic nanocrystals: Syntheses, properties, and applications. Chem. Rev. 116, 10414-10472 (2016)

15. Alayoglu, S., Nilekar, A. U., Mavrikakis, M. \& Eichhorn, B. Ru-Pt core-shell nanoparticles for preferential oxidation of carbon monoxide in hydrogen. Nat. Mater. 7, 333-338 (2008).

16. Wang, D. et al. Structurally ordered intermetallic platinum-cobalt core-shell nanoparticles with enhanced activity and stability as oxygen reduction electrocatalysts. Nat. Mater. 12, 81-87 (2013).

17. Shao, M., Chang, Q., Dodelet, J. -P. \& Chenitz, R. Recent advances in electrocatalysts for oxygen reduction reaction. Chem. Rev. 116, 3594-3657 (2016).

18. Musselwhite, N. et al. Isomerization of $n$-hexane catalyzed by supported monodisperse PtRh bimetallic nanoparticles. Catal. Lett. 143, 907-911 (2013).

19. Zafeiratos, S., Piccinin, S. \& Teschner, D. Alloys in catalysis: phase separation and surface segregation phenomena in response to the reactive environment. Catal. Sci. Technol. 2, 1787-1801 (2012).

20. Tao, F. et al. Reaction-driven restructuring of Rh-Pd and Pt-Pd core-shell nanoparticles. Science 322, 932-934 (2008).

21. Liao, H., Fisher, A. \& Xu, Z. J. Surface segregation in bimetallic nanoparticles: A critical issue in electrocatalyst engineering. Small 11, 3221-3246 (2015).

22. Fu, Q., Yang, F. \& Bao, X. Interface-confined oxide nanostructures for catalytic oxidation reactions. Acc. Chem. Res. 46, 1692-1701 (2013)

23. Xu, X., Fu, Q., Wei, M., Wu, X. \& Bao, X. Comparative studies of redox behaviors of $\mathrm{Pt}-\mathrm{Co} / \mathrm{SiO}_{2}$ and $\mathrm{Au}-\mathrm{Co} / \mathrm{SiO}_{2}$ catalysts and their activities in CO oxidation. Catal. Sci. Technol. 4, 3151-3158 (2014).

24. Wang, G. -H. et al. Platinum-cobalt bimetallic nanoparticles in hollow carbon nanospheres for hydrogenolysis of 5-hydroxymethylfurfural. Nat. Mater. 13, 293-300 (2014).

25. Luo, J. et al. Mechanisms for high selectivity in the hydrodeoxygenation of 5-hydroxymethylfurfural over PtCo nanocrystals. ACS Catal. 6, 4095-4104 (2016).

26. Ha, D.-H., Moreau, L. M., Honrao, S., Hennig, R. G. \& Robinson, R. D. The oxidation of cobalt nanoparticles into Kirkendall-hollowed $\mathrm{CoO}$ and $\mathrm{Co}_{3} \mathrm{O}_{4}$ : The diffusion mechanisms and atomic structural transformations. J. Phys. Chem. C. 117, 14303-14312 (2013).

27. Papaefthimiou, V. et al. When a metastable oxide stabilizes at the nanoscale: wurtzite $\mathrm{CoO}$ formation upon dealloying of PtCo nanoparticles. J. Phys. Chem. Lett. 2, 900-904 (2011).

28. Xin, H. L. et al. Revealing the atomic restructuring of Pt-Co nanoparticles. Nano Lett. 14, 3203-3207 (2014).

29. $\mathrm{Fu}, \mathrm{Q}$. et al. Interface-confined ferrous centers for catalytic oxidation. Science 328, 1141-1144 (2010).

30. Ning, Y. et al. Nature of interface confinement effect in oxide/metal catalysts. J. Phys. Chem. C. 119, 27556-27561 (2015).

31. Dai, S. et al. Revealing surface elemental composition and dynamic processes involved in facet-dependent oxidation of $\mathrm{Pt}_{3} \mathrm{Co}$ nanoparticles via in situ transmission electron microscopy. Nano Lett. 17, 4683-4688 (2017).

32. Netzer, F. P., Allegretti, F. \& Surnev, S. Low-dimensional oxide nanostructures on metals: Hybrid systems with novel properties. J. Vac. Sci. Technol. B 28, 1-16 (2010).

33. Prada, S., Martinez, U. \& Pacchioni, G. Work function changes induced by deposition of ultrathin dielectric films on metals: A theoretical analysis. Phys. Rev. B 78, 235423 (2008).

34. Michaelides, A. \& Hu, P. Catalytic water formation on platinum: A firstprinciples study. J. Am. Chem. Soc. 123, 4235-4242 (2001).

35. Sun, D. et al. Theoretical study of the role of a metal-cation ensemble at the oxide-metal boundary on CO oxidation. J. Phys. Chem. C. 116, 7491-7498 (2012).

36. Qadir, K. et al. Tailoring metal-oxide interfaces of inverse catalysts of $\mathrm{TiO}_{2} /$ nanoporous-Au under hydrogen oxidation. Chem. Commun. 51, 9620-9623 (2015).

37. Green, I. X., Tang, W., Neurock, M. \& Yates, J. T. Low-temperature catalytic $\mathrm{H}_{2}$ oxidation over Au nanoparticle/TiO 2 dual perimeter sites. Angew. Chem. Int. Ed. 50, 10186-10189 (2011).

38. Saavedra, J., Doan, H. A., Pursell, C. J., Grabow, L. C. \& Chandler, B. D. The critical role of water at the gold-titania interface in catalytic $\mathrm{CO}$ oxidation. Science 345, 1599-1602 (2014).

39. Kresse, G. \& Furthmüller, J. Efficient iterative schemes for ab initio totalenergy calculations using a plane-wave basis set. Phys. Rev. B 54, 11169 (1996).

40. Kresse, G. \& Hafner, J. Ab initio molecular dynamics for liquid metals. Phys. Rev. B 47, 558 (1993).

41. Kresse, G. \& Hafner, J. Ab initio molecular-dynamics simulation of the liquidmetal-amorphous-semiconductor transition in germanium. Phys. Rev. B 49, 14251 (1994).
42. Kresse, G. \& Joubert, D. From ultrasoft pseudopotentials to the projector augmented-wave method. Phys. Rev. B 59, 1758 (1999).

43. Grimme, S., Antony, J., Ehrlich, S. \& Krieg, H. A consistent and accurate ab initio parametrization of density functional dispersion correction (DFT-D) for the 94 elements H-Pu. J. Chem. Phys. 132, 154104 (2010).

44. Hammer, B., Hansen, L. B. \& Nørskov, J. K. Improved adsorption energetics within density-functional theory using revised Perdew-Burke-Ernzerhof functionals. Phys. Rev. B 59, 7413 (1999).

45. Perdew, J. P., Burke, K. \& Ernzerhof, M. Generalized gradient approximation made simple. Phys. Rev. Lett. 77, 3865 (1996).

46. Blöchl, P. E. Projector augmented-wave method. Phys. Rev. B 50, 17953 (1994).

47. Henkelman, G., Uberuaga, B. P. \& Jónsson, H. A climbing image nudged elastic band method for finding saddle points and minimum energy paths. $J$. Chem. Phys. 113, 9901-9904 (2000).

48. Henkelman, G. \& Jónsson, H. Improved tangent estimate in the nudged elastic band method for finding minimum energy paths and saddle points. J. Chem. Phys. 113, 9978-9985 (2000).

49. Monkhorst, H. J. \& Pack, J. D. Special points for Brillouin-zone integrations. Phys. Rev. B 13, 5188 (1976).

\section{Acknowledgements}

This work was supported by the Institute for Basic Science (IBS) [IBS-R004]. K. An thanks the Basic Science Research Program through the National Research Foundation of Korea (NRF) funded by the Ministry of Education (2015R1C1A1A01055092). Y. Jung acknowledges support through the National Research Foundation of Korea from the Korean Government (NRF-2017R1A2B3010176, NRF-2016M3D1A1021147).

\section{Authors contributions}

H.L. and J.Y.P. designed and the experiments. H.L. carried out the central measurements and analysis presented in this work. C.L. and H. L. fabricated the devices. K.A. and H.L. carried out the synthesis experiments. J.W.S., H.L., and R.R. performed the in situ TEM experiments. J.L., S.B., and Y.J. performed the DFT calculations. H.L., J.L., Y.J., and J.Y.P. wrote the manuscript and all authors edited the final manuscript.

Reprints and permission information is available online at http://npg.nature.com/ reprintsandpermissions/

Publisher's note: Springer Nature remains neutral with regard to jurisdictional claims in published maps and institutional affiliations.

\section{Additional information}

Supplementary Information accompanies this paper at https://doi.org/10.1038/s41467018-04713-8.

Competing interests: The authors declare no competing interests.

Reprints and permission information is available online at http://npg.nature.com/ reprintsandpermissions/

Publisher's note: Springer Nature remains neutral with regard to jurisdictional claims in published maps and institutional affiliations.

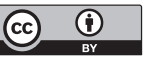

Open Access This article is licensed under a Creative Commons Attribution 4.0 International License, which permits use, sharing, adaptation, distribution and reproduction in any medium or format, as long as you give appropriate credit to the original author(s) and the source, provide a link to the Creative Commons license, and indicate if changes were made. The images or other third party material in this article are included in the article's Creative Commons license, unless indicated otherwise in a credit line to the material. If material is not included in the article's Creative Commons license and your intended use is not permitted by statutory regulation or exceeds the permitted use, you will need to obtain permission directly from the copyright holder. To view a copy of this license, visit http://creativecommons.org/ licenses/by/4.0/.

(C) The Author(s) 2018 